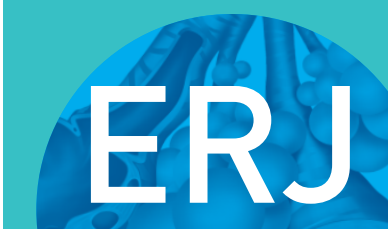

open research
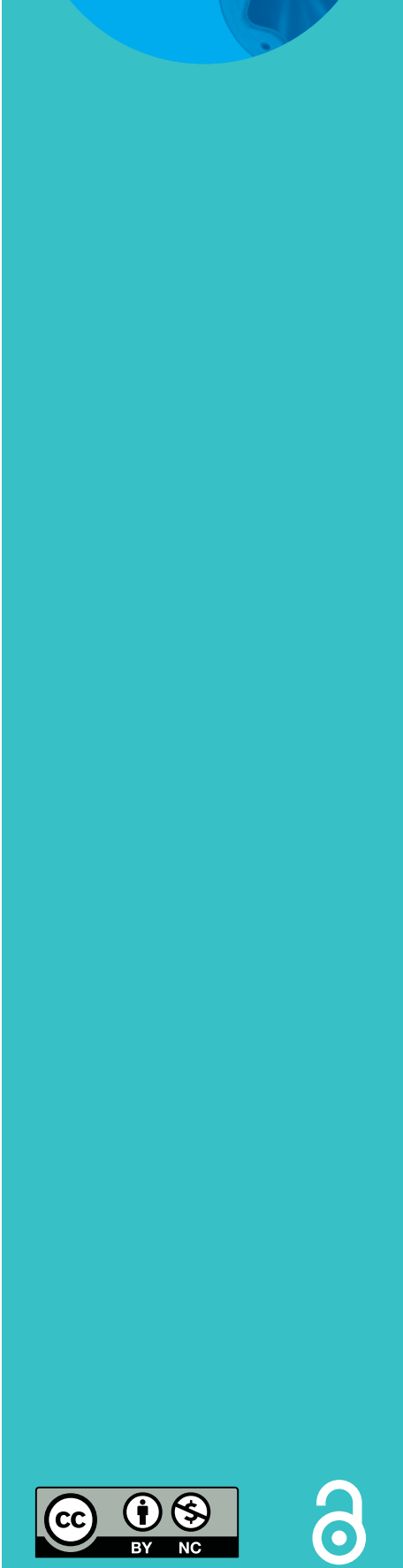

\section{ERS International Congress 2020: highlights from the General Pneumology Assembly}

\author{
Luke Daines (10 ${ }^{1}$, Joren Buekers (10 ${ }^{2,3,4,5}$, Beatriz Abascal Bolado ${ }^{6}$, \\ Janwillem W.H. Kocks ${ }^{7,8,9}$, Guido Vagheggini (10) ${ }^{10,11}$, Vitalii Poberezhets ${ }^{12}$, \\ loannis Vogiatzis ${ }^{13}$, Hilary Pinnock ${ }^{1}$ and Lowie E.G.W. Vanfleteren (id ${ }^{14,15}$
}

Affiliations: ${ }^{1}$ Allergy and Respiratory Research Group, Usher Institute, The University of Edinburgh, Edinburgh, UK. ${ }^{2}$ ISGlobal, Barcelona, Spain. ${ }^{3}$ Universitat Pompeu Fabra (UPF), Barcelona, Spain. ${ }^{4} \mathrm{CIBER}$ Epidemiología y Salud Pública (CIBERESP), Barcelona, Spain. ${ }^{5}$ Measure, Model and Manage Bioresponses (M3-BIORES), Dept of Biosystems, KU Leuven, Leuven, Belgium. ${ }^{6}$ Pneumology Dept, Hospital Universitario Marqués de Valdecilla, Santander, Spain. ${ }^{7}$ General Practitioners Research Institute, Groningen, The Netherlands. ${ }^{8}$ GRIAC Research Institute, University Medical Center Groningen, University of Groningen, Groningen, The Netherlands. ${ }^{9}$ Observational and Pragmatic Research Institute, Singapore. ${ }^{10}$ Chronic Respiratory Failure Care Pathway, Dept of Medical Specialties, Azienda USL Toscana Nordovest, Pisa, Italy. ${ }^{11}$ Fondazione Volterra Ricerche ONLUS, Volterra, Italy. ${ }^{12}$ Dept of Propedeutics of Internal Medicine, National Pirogov Memorial Medical University, Vinnytsya, Ukraine. ${ }^{13}$ Faculty of Health and Life Sciences, Northumbria University Newcastle, Newcastle upon Tyne, UK. ${ }^{14}$ COPD Center, Dept of Respiratory Medicine and Allergology, Sahlgrenska University Hospital, Gothenburg, Sweden. ${ }^{15}$ Dept of Internal Medicine and Clinical Nutrition, Institute of Medicine, Sahlgrenska Academy, University of Gothenburg, Gothenburg, Sweden.

Correspondence: Lowie E.G.W. Vanfleteren, Dept of Respiratory Medicine and Allergology, Sahlgrenska University Hospital, Vita Stråket 12, 41345 Gothenburg, Sweden. E-mail: lowie.vanfleterenđavgregion.se

ABSTRACT The European Respiratory Society (ERS) accepted 4062 abstracts for presentation at the ERS International Congress in 2020. Although the conference was held virtually, making it possible to replay presentations, it remains a challenge to keep abreast of all the clinical and scientific advances. Therefore, this article provides highlights from the General Pneumology Assembly. Selected presentations from rehabilitation and chronic care, general practice and primary care, and electronic/mobile health (e-health/ $\mathrm{m}$-health) are summarised. The highlights incorporate novel findings from laboratory-based science, randomised controlled trials and qualitative research together with insights from newly available clinical guidelines.

@ERSpublications

Highlights from the @EuroRespSoc General Pneumology Assembly presented during \#ERSCongress 2020 https://bit.ly/369M3J2

Cite this article as: Daines L, Buekers J, Bolado BA, et al. ERS International Congress 2020: highlights from the General Pneumology Assembly. ERJ Open Res 2021; 7: 00841-2020 [https:// doi.org/10.1183/23120541.00841-2020].

Copyright $\odot$ ERS 2021. This article is open access and distributed under the terms of the Creative Commons Attribution Non-Commercial Licence 4.0. 


\section{Introduction}

With travel restrictions and social distancing the norm for much of the year due to coronavirus disease (COVID-19), the European Respiratory Society (ERS) International Congress, held virtually in September 2020, provided a much valued opportunity to come together. As the world's largest scientific and educational conference for respiratory medicine, the ERS International Congress provided an excellent chance to hear the latest developments in research and clinical practice with 4062 abstracts accepted for presentation. The General Pneumology Assembly is the largest of the 14 Assemblies within the ERS. Across the four groups within the Assembly, 346 abstracts were presented in 22 sessions. Although the virtual platform allowed presentations to be replayed, it can be difficult to keep up to date with all of the scientific and clinical advances on offer. Therefore, in this article we aim to share some of the highlights arising from the General Pneumology Assembly.

\section{Pulmonary rehabilitation and chronic care}

Session: Best abstracts in pulmonary rehabilitation and chronic care

A peer review of abstracts submitted to the ERS Congress in the field of pulmonary rehabilitation and chronic care led to the selection of the six most innovative and interesting works, presented by their authors in the traditional session "Best abstracts in pulmonary rehabilitation and chronic care". Works selected this year covered key topics, including cellular mechanisms of muscle regeneration, effects of high-flow oxygen supplementation during exercise in chronic obstructive pulmonary disease (COPD) and interstitial lung disease, the efficacy of new exercise training strategies for COPD patients, and effects of a behavioural change intervention on asthma symptoms.

To investigate the cellular basis of reduced efficacy of exercise training in peripheral muscles in COPD patients, POMIĖs et al. [1] used in vitro electrical pulse stimulation (EPS) of cultured human myotubes from nine healthy subjects and nine COPD patients. Compared with myotubes from healthy subjects, after a 24-h EPS protocol at $1 \mathrm{~Hz}$ and $11.5 \mathrm{~V}$, myotubes from COPD patients appeared atrophied, had reduced cellular differentiation and regeneration, and showed impaired protein synthesis and oxidative metabolism adaptation. This in vitro altered response to EPS could reflect impaired regenerative capacities of satellite cells, accounting for a lack of muscle adaptation capacity and the limited effects of in vivo exercise training in COPD patients.

In the same research area of muscle repair and regeneration, SANCHO MUÑOZ et al. [2] investigated the role of the negative regulator myostatin as a possible inhibitor of muscle regeneration in sarcopenic COPD patients. Compared with control subjects and COPD patients with normal body composition, sarcopenic COPD patients had a reduction in quadriceps muscle strength, higher myostatin protein levels, and increased activated satellite cells, hybrid fibres and TUNEL-positive cell counts. Muscle fibre area and expression of muscle regeneration markers were also lower than in controls. The authors suggested that an increased expression of myostatin may have interfered with muscle cell proliferation during the regeneration process in COPD patients with sarcopenia, thus leading to poor muscle growth following injury and muscle mass loss.

The effects of high-flow nasal cannula (HFNC) for oxygen supplementation during exercise were analysed in two selected abstracts. Generating high humidified airflow with constant oxygen fraction, HFNC allows for a decrease in oxygen dilution in the dead space of the airway. Thus, HFNC generates positive pressure, improves oxygenation, reduces the respiratory rate and contributes to greater comfort in acute respiratory failure [3]. In a randomised crossover study, SANGUANWONG et al. [4] compared the effects of supplemental HFNC, oxygen cannula and without oxygen (control group) during exercise in 13 COPD patients with exercise-induced desaturation. Subjects were assigned to wear HFNC $\left(60 \mathrm{~L} \cdot \mathrm{min}^{-1}\right.$, inspiratory oxygen fraction $\left(F_{\mathrm{IO}_{2}}\right)$ 0.4), oxygen cannula $\left(6 \mathrm{~L} \cdot \mathrm{min}^{-1}\right)$ or no oxygen supplement during a cycle ergometer constant work rate exercise test, set at $70 \%$ of peak work rate. Endurance exercise time, dyspnoea and time to desaturation improved with HFNC and oxygen cannula, with no superiority of HFNC compared with oxygen cannula. ARIzono et al. [5] assessed the effect of HFNC therapy on exercise capacity in 20 patients following an exacerbation of interstitial lung disease. This prospective, single-blind, randomised trial compared the efficacy during exercise of two different concentrations of oxygen for $\mathrm{HFNC}$ : high $\left(F_{\mathrm{IO}_{2}}\right.$ $\left.60 \%, 40 \mathrm{~L} \cdot \mathrm{min}^{-1}\right)$ and low $\left(F_{\mathrm{IO}_{2}}, 25.1 \pm 6.7 \%, 40 \mathrm{~L} \cdot \mathrm{min}^{-1}\right)$ oxygen concentration. During a constant work rate exercise test $(20 \mathrm{~W}) 9$ days after the exacerbation, with high oxygen concentration, HFNC significantly increased endurance time and minimum oxygen saturation while heart rate and respiratory rate were decreased compared with low oxygen, suggesting a high concentration of oxygen for HFNC benefits exercise capacity.

One of the most relevant challenges in pulmonary rehabilitation and chronic care is how to induce a sustained change in a patient's lifestyle to maintain the beneficial effects of pulmonary rehabilitation and improve control of symptoms and quality of life. Because improvement in physical activity levels and sleep 
quality may be related to asthma control in adults, PAssos et al. [6] conducted a randomised controlled trial to investigate whether a behavioural change intervention could improve physical activity levels and sleep quality in moderate to severe asthma patients. The intervention group followed the same usual care and educational programme of the control group, but with the addition of an 8-week behavioural change intervention focused on goal setting and positive feedback. All subjects were assessed on sleep quality (ActiSleep, Pittsburgh and Berlin questionnaires), physical activity levels (ActiGraph GT9X accelerometer), asthma symptoms (daily diary) and psychosocial symptoms. A significant improvement in the intervention group was observed in sleep efficiency and latency, depression symptoms, physical activity levels, and the number of asthma symptom-free days compared with the control group. The authors suggested that beneficial effects of the behavioural change intervention on asthma control and psychosocial symptoms were mediated by the increase in physical activity levels and sleep quality.

To increase adherence to pulmonary rehabilitation programmes, alternative approaches to conventional programmes have been proposed, focusing on group activities and participation in immersive and engaging social and physical activity. Singing for Lung Health was recently proposed by the British Lung Foundation as a group activity tailored to people with respiratory disease (particularly those with airflow obstruction), and focused on improving breath control and posture using songs [7]. Lung choirs have been shown to improve respiratory control and wellbeing, but the impact on physical capacity was unclear. KAASGAARD et al. [8] conducted a multicentre randomised controlled trial to evaluate the effectiveness of singing training in increasing physical capacity compared with usual physical training. Singing training was as efficacious as physical training in improving physical capacity and superior for improving quality of life. Although this study confirmed singing training as a promising strategy for physical training, no differences were found in symptom control or adherence, suggesting the need for further efforts to standardise the content of singing training to provide evidence-based recommendations.

\section{General practice and primary care}

\section{Session: Primary care day and diagnosis and management of airways disease in primary care}

The ERS International Congress 2020 offered much for participants interested in primary care respiratory medicine. Highlights from the primary care day and the diagnosis and management of airways disease in primary care sessions are presented.

Diagnosis of asthma or COPD can be difficult and has been more challenging during the COVID-19 pandemic. As a heterogenous disease with different phenotypes [9] and no "gold standard" test that can confirm or refute the diagnosis in every situation, misdiagnosis of asthma is common [10, 11]. Mike Thomas emphasised the importance of gaining objective evidence for an asthma diagnosis rather than relying on symptoms alone. In COPD, spirometry is the key diagnostic test [12], but as Miguel Roman Rodriguez highlighted, there are barriers to confirming the diagnosis, meaning many cases of COPD remain undiagnosed [13]. COVID-19 has bought new challenges for diagnostic assessment, principally due to reduced lung physiology services in many countries [14]. Until laboratory-based testing becomes available again, alternative options might include peak expiratory flow, portable spirometers and exhaled nitric oxide monitors, and smartphone technologies [14].

In asthma management, overuse of short-acting $\beta_{2}$-agonist (SABA) inhalers is associated with poor clinical outcomes [9], including an increased risk of asthma attacks, emergency department visits [15] and mortality [16]. In their study of 70 patients who presented to the emergency department with an asthma attack, BARMPARESSOU et al. [17] identified that most individuals had "mild" asthma and 27\% were treated only with a SABA. To reduce SABA overprescribing in the UK, electronic prescribing alerts were introduced in general practices. McKibBen et al. [18] found a clear discrepancy between how clinicians, experts and guidelines defined excessive SABA use. The volume of warnings and other work pressures meant that SABA alerts were often ignored [18].

One intervention known to reduce emergency department attendances is supported self-management for asthma [19]. Asthma action plans can be effectively tailored for different cultural groups [19], but the influence of limited health literacy on self-management needs further exploration. By conducting a qualitative study of 26 participants recruited from Malaysian primary care, SALIM et al. [20] found poor understanding of asthma led to limited acceptance of the condition and treatment, resulting in poor asthma control. Supporting people with limited health literacy to understand their condition was recommended for developing good self-management [20].

Helping women with asthma during pregnancy was discussed by Vibeke Backer who highlighted guidance from the ERS/Thoracic Society of Australia and New Zealand task force [21]. In comparison with nonasthmatic women, women with asthma have on average a longer time to pregnancy and become pregnant less often [22]. When considering asthma medication use during pregnancy, inhaled therapies, 
oral corticosteroids and most antihistamines were considered to be without serious side-effects [21]. The main aim should be to prevent exacerbations, as events which lead to lower maternal oxygenation are more harmful for the (unborn) child than the potential side-effects of medication. Currently, few studies are available to inform the use of biological therapies during pregnancy; however, monoclonal antibodies are considered unlikely to cause harm to the fetus and on balance should be continued during pregnancy if needed to control the mother's asthma [21].

The remaining talks considered how primary care clinicians can best support individuals with respiratory disease that often results from, or coexists with, other conditions. Individuals with COPD often have multiple comorbidities affecting their health and prognosis [12, 23]. Comorbidities in COPD often occur in clusters, which suggests common risk factors and shared disease processes [24]. Ioanna Tsiligianni highlighted the International Primary Care Respiratory Group desktop helper for COPD and multimorbidity, recommending a holistic approach: identifying and treating comorbidities, screening for coexisting anxiety or depression, and evaluating the indications and possible side-effects of inhaled therapies [25]. One important comorbidity of COPD which carries a poor prognosis is pulmonary hypertension [26]. Defined by a mean pulmonary arterial pressure $>25 \mathrm{mmHg}$, pulmonary hypertension may arise as a complication of many cardiovascular and respiratory diseases, but can be difficult to identify as right heart failure occurs in the later stages of disease [26]. To achieve a timely diagnosis (and better clinical outcomes), Anton Vonk Noordegraaf recommended being alert to the primary symptom of exercise intolerance, especially in younger patients (aged 30-55 years), and instigating further assessment with N-terminal pro-brain natriuretic peptide, ECG and echocardiography [26].

Despite the restrictions due to COVID-19, the virtual ERS International Congress 2020 provided opportunities to learn, discuss and share research and experiences from across the world, and connect with one another, making the primary care sessions this year particularly valuable and stimulating.

\section{m-Health/e-health}

Session: Digital technologies in airway diseases

Growing interest of the ERS in digital medicine interventions resulted in an oral presentation session organised by Group 1.4 (m-Health/e-Health) dedicated to the use of modern technologies in the management and treatment of airway diseases. In this rapidly changing world, healthcare workers are facing new challenges: How can we use new technologies to improve our healthcare system? How can we ensure that technologies are adopted and useable rather than leaving people baffled? At the same time, respiratory patients are extremely interested in how they can optimally benefit from innovative technologies. The session "Digital technologies in airway diseases" provided participants with different solutions to these questions.

\section{Feedback from patients}

When dealing with digital technologies, it is always important to put the needs and preferences of patients first. In this regard, Khusial et al. [27] and MetTing and LaHr [28] reported on feedback they gathered from patients who had tried digital monitoring systems. KHUSIAL et al. [27] organised focus groups for patients with asthma to explore their attitudes towards $\mathrm{m}$-health applications. Improving disease outcomes, reducing consultations and improving disease awareness were all identified as potential goals. Ineffectiveness, time consumption, privacy issues and information overload were the most important perceived barriers. Interestingly, patients had divergent opinions on the required functions of an m-health system, suggesting that these systems should be personalised to their individual needs. METTING and LAHR [28] developed a monitoring system in the Horizon 2020 Connecare study to coordinate care around chronically ill patients. The system consisted of a dashboard for healthcare providers and an app for patients. The authors elaborated on the lessons learned from the feedback provided by 46 COPD patients. Most of these patients highlighted the ease of use of the chat function. In addition, the authors emphasised the importance of personalisation and including all stakeholders in the development of a monitoring system.

\section{Remote healthcare}

Telemonitoring of vital signs and parameters from patients is a well-known way to use digital technologies. However, telemonitoring could also be used for helping patients at a distance. Cumella et al. [29] showed that a WhatsApp (www.whatsapp.com) service can reach an otherwise unengaged younger audience of asthma patients. Patients could reach healthcare providers through WhatsApp and, in response, they received advice, links, video content or infographics from healthcare providers. A post-chat online survey of 669 patients with asthma showed the service was well perceived and almost two-thirds of the patients had more confidence in managing their asthma symptoms after using the service. ADEJUMO et al. [30] performed a randomised controlled pilot study with 36 asthma patients to assess whether feedback about inhaler use could affect adherence and clinical outcomes. Inhaler use was monitored with an electronic 
monitoring device for both intervention and control groups. Only the intervention group received monthly feedback and an app showing their day-to-day inhaler use. A trend towards higher adherence for the intervention group was identified, whereas no changes in clinical outcomes were observed.

\section{Exacerbation prediction}

One of the most common goals of digital technologies for patients with chronic airway diseases is the prediction of exacerbations and the reduction of hospitalisations. JANSON et al. [31] developed a machine learning model to predict the immediate risk of asthma exacerbations. Data from electronic medical records and national registries of almost 30000 asthma patients were used to predict whether they would exacerbate in the next 15 days. The model indicated that the Charlson Comorbidity Index and previous exacerbations were the main factors influencing the risk of exacerbations in these patients, but was not sufficiently accurate to be used in clinical practice. Therefore, the authors concluded that supplemental data (e.g. pollution, pollen count and information from $\mathrm{m}$-health devices) were needed to develop a more clinically useful tool. The e-health intervention (EmmaCOPD; https://ec.europa.eu/eip/ageing/repository/ emmacopd_en) developed by VAN BUUL et al. [32] managed to reduce the total number of exacerbations and hospitalisation days among COPD patients. EmmaCOPD consisted of an app to answer symptom questionnaires and a smartwatch with a step counter. Depending on the answers to the questionnaires, the 29 included patients with COPD were assigned to a green, yellow, orange or red zone. The system provided patients with the advice to call a healthcare provider when they entered the orange or red zone. As a result, EmmaCOPD reduced the total number of exacerbations and hospitalisation days, while additional work for healthcare providers was kept to a minimum.

Overall, the session highlighted the most important cases of digital technologies for patients with airway diseases from different countries and improved our understanding of how we should build future healthcare systems.

\section{Concluding remarks}

The General Pneumology Assembly of the ERS offers a wide range of clinical and scientific research, as is evident in this summary. Given the size of the ERS International Congress, it can be hard to keep abreast of new developments, even using a virtual platform. Therefore, we hope the highlights from the Assembly summarised in this article will allow readers to keep up to date with the latest research, revisit topics of interest and provide encouragement to take part in the ERS International Congress in 2021 to be held in Barcelona.

Conflict of interest: L. Daines has nothing to disclose. J. Buekers reports grants from European Respiratory Society (ERS Long-Term Research Fellowship 2020), and other support from the Spanish Ministry of Science and Innovation through the "Centro de Excelencia Severo Ochoa 2019-2023" Program (CEX2018-000806-S) and the Generalitat de Catalunya through the CERCA Program, during the conduct of the study. B. Abascal Bolado has nothing to disclose. J.W.H. Kocks reports grants, personal fees and nonfinancial support from AstraZeneca and Boehringer Ingelheim, grants and personal fees from Chiesi Pharmaceuticals, grants, personal fees and nonfinancial support from GSK, grants and personal fees from Novartis, and grants from Mundipharma and TEVA, outside the submitted work; and $72.5 \%$ of shares in the General Practitioners Research Institute. G. Vagheggini has nothing to disclose. V. Poberezhets has nothing to disclose I. Vogiatzis has nothing to disclose. H. Pinnock has nothing to disclose. L.E.G.W. Vanfleteren reports grants and personal fees from AstraZeneca, and personal fees from Novartis, GSK, Chiesi, Menarini, Pulmonx, ResMed, Boehringer, Verona Pharma and AGA Linde, outside the submitted work.

Support statement: J. Buekers acknowledges support from the European Respiratory Society Long-Term Research Fellowship 2020, the Spanish Ministry of Science and Innovation through the "Centro de Excelencia Severo Ochoa 2019-2023" Program (CEX2018-000806-S), and the Generalitat de Catalunya through the CERCA Program.

\section{References}

1 Pomiès $\mathrm{P}$, Catteau $\mathrm{M}$, Passerieux E, et al. In vitro electrostimulation reveals a lack of muscle adaptation in myotubes from COPD patients. Eur Respir J 2020; 56: Suppl. 64, 4658

2 Sancho Muñoz A, Guitart M, Rodríguez D, et al. Increased myostatin as a negative regulator of muscle regeneration potential in sarcopenic COPD patients: clinical implications. Eur Respir J 2020; 56: Suppl. 64, 4659.

3 Frat JP, Thille AW, Mercat A, et al. High-flow oxygen through nasal cannula in acute hypoxemic respiratory failure. N Engl J Med 2015; 372: 2185-2196.

4 Sanguanwong N, Sae-Eao N, Ananpipatkul A, et al. Effects of high flow nasal cannula during exercise in COPD patients with exercise induced desaturation. Eur Respir J 2020; 56: Suppl. 64, 4661.

5 Arizono S, Oomagari M, Yanagita Y, et al. Benefits of high-flow nasal cannula oxygen therapy on exercise capacity following acute exacerbation in ILD patients. Eur Respir J 2020; 56: Suppl. 64, 4662.

6 Passos NFP, Carvalho-Pinto RM, Cukier A, et al. Effects of a behaviour change intervention aimed to increase physical activity on sleep quality of adults with asthma: an RCT. Eur Respir J 2020; 56: Suppl. 64, 4660.

7 Lewis A, Cave P, Stern M, et al. Singing for lung health - a systematic review of the literature and consensus statement. NPJ Prim Care Respir Med 2016; 26: 16080. 
8 Kaasgaard M, Rasmussen DB, Løkke Ottesen A, et al. Sing-a-Lung: group singing as training modality in pulmonary rehabilitation for patients with chronic obstructive pulmonary disease (COPD): a multicenter, cluster-randomised, non-inferiority controlled trial. Eur Respir J 2020; 56: Suppl. 64, 4663.

9 Global Initiative for Asthma. Global Strategy for Asthma Management and Prevention. 2020. www.ginasthma.org/ wp-content/uploads/2020/04/GINA-2020-full-report_-final-_wms.pdf Date last accessed: September 1, 2020.

10 Aaron SD, Vandemheen KL, FitzGerald JM, et al. Reevaluation of diagnosis in adults with physician-diagnosed asthma. JAMA 2017; 317: 269-279.

11 Looijmans-Van den Akker I, van Luijn K, Verheij T. Overdiagnosis of asthma in children in primary care: a retrospective analysis. Br J Gen Pract 2016; 66: e152-e157.

12 Global Initiative for Chronic Obstructive Lung Disease. Global Strategy for the Diagnosis, Management, and Prevention of Chronic Obstructive Pulmonary Disease. 2020. www.goldcopd.org/wp-content/uploads/2019/11/ GOLD-2020-REPORT-ver1.0wms.pdf Date last accessed: September 1, 2020.

13 Soriano JB, Zielinski J, Price D. Screening for and early detection of chronic obstructive pulmonary disease. Lancet 2009; 374: 721-732.

14 Kouri A, Gupta S, Yadollahi A, et al. CHEST reviews: addressing reduced laboratory-based pulmonary function testing during a pandemic. Chest 2020; 158: 2502-2510.

15 Stanford RH, Shah $\mathrm{MB}$, D'Souza AO, et al. Short-acting $\beta$-agonist use and its ability to predict future asthma-related outcomes. Ann Allergy Asthma Immunol 2012; 109: 403-407.

16 Suissa S, Ernst P, Boivin JF, et al. A cohort analysis of excess mortality in asthma and the use of inhaled beta-agonists. Am J Respir Crit Care Med 1994; 149: 604-610.

17 Barmparessou Z, Korkontzelou A, Ioannou M, et al. The majority of patients that suffer an asthma attack at the Emergency Room (ER) are mild asthmatics. Eur Respir J 2020; 56: Suppl. 64, 4012.

18 McKibben S, Bush A, Thomas M, et al. "I don't think there's a simple way of doing it": the use of an electronic

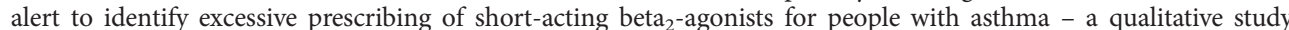
with asthma experts and primary care staff. Eur Respir J 2020; 56: Suppl. 64, 4013.

19 Pinnock H, Parke HL, Panagioti M, et al. Systematic meta-review of supported self-management for asthma: a healthcare perspective. BMC Med 2017; 15: 64 .

20 Salim HS, Young I, Ghazali SS, et al. Negotiating identity: impact on self-management practices in people with asthma and limited health literacy in Malaysia. Eur Respir J 2020; 56: Suppl. 64, 4014.

21 Middleton PG, Gade EJ, Aguilera C, et al. ERS/TSANZ Task Force Statement on the management of reproduction and pregnancy in women with airways diseases. Eur Respir J 2020; 55: 1901208.

22 Gade EJ, Thomsen SF, Lindenberg S, et al. Fertility outcomes in asthma: a clinical study of 245 women with unexplained infertility. Eur Respir J 2016; 47: 1144-1151.

23 Divo M, Cote C, de Torres JP, et al. Comorbidities and risk of mortality in patients with chronic obstructive pulmonary disease. Am J Respir Crit Care Med 2012; 186: 155-161.

24 Divo MJ, Casanova C, Marin JM, et al. COPD comorbidities network. Eur Respir J 2015; 46: 640-650.

25 Tsiligianni I, Hoines K, Jensen C, et al. Desktop Helper No. 10 - Rational use of inhaled medications for the patient with COPD and multiple comorbid conditions: guidance for primary care. 2019. www.ipcrg.org/dth10 Date last accessed: October 1, 2020.

26 Galiè N, Humbert M, Vachiery JL, et al. ESC/ERS guidelines for the diagnosis and treatment of pulmonary hypertension: the joint task force for the diagnosis and treatment of pulmonary hypertension of the European Society of Cardiology (ESC) and the European Respiratory Society (ERS) endorsed by: Association for European Paediatric and Congenital Cardiology (AEPC), International Society for Heart and Lung Transplantation (ISHLT). Eur Respir J 2015; 46: 903-975.

27 Khusial R, Van Koppen S, Honkoop P, et al. Davos@home: patient and health care providers' perceptions on mHealth after high altitude climate treatment. Eur Respir J 2020; 56: Suppl. 64, 4801.

28 Metting EI, Lahr MMH. Promising integrated care platform developed with and tested by respiratory patients: lessons learned form the European H2020 Connecare study. Eur Respir J 2020; 56: Suppl. 64, 4800.

29 Cumella A, King J, Sinton H, et al. Engaging younger people in their asthma management - findings from a pilot telemedicine asthma nurse service. Eur Respir J 2020; 56: Suppl. 64, 4805.

30 Adejumo I, Patel M, Mckeever TM, et al. Feedback on inhaler use does not significantly improve inhaled corticosteroid adherence or clinical outcomes. Eur Respir J 2020; 56: Suppl. 64, 4804.

31 Janson C, Johansson G, Larsson K, et al. Use of machine learning to predict asthma exacerbations. Eur Respir J 2020; 56: Suppl. 64, 4802.

32 Van Buul A, Voeten R, Derksen-Franken C, et al. An eHealth program to reduce hospitalizations due to acute exacerbation of chronic obstructive pulmonary disease - a real-life study. Eur Respir J 2020; 56: Suppl. 64, 4799. 